\title{
Spatial Cues in Small Screen Devices: Benefit Or Handicap?
}

\author{
Martina Ziefle \\ Human Technology Centre (HUMTEC) Theaterplatz 14, \\ RWTH Aachen University, 52062 Aachen, Germany \\ ziefle@humtec.rwth-aachen.de
}

\begin{abstract}
Disorientation in small-screen-device menus is a frequent problem for many users. This study examines if navigation aids containing spatial information may reduce disorientation. Two aids were implemented into a simulated mobile phone: One aid contained spatial cues, providing an overview of menu locations and routes, the other delivered landmark knowledge, representing salient features on the route. Also, a condition without any aid was examined. 24 children and 24 adults solved four phone tasks twice. Dependent variables were navigation effectiveness and efficiency. Performance was lowest when users were not supported by any aid. The aid providing survey knowledge yielded the best performance. Though, the relative benefit by aids revealed to be age-related. While the aid providing survey knowledge was advantageous for all users, the aid delivering landmark knowledge was especially harmful for the children, which showed considerably more detouring in the menu. It is concluded that navigation aids reduce disorientation in small devices, especially those which support users to build up a spatial representation of the menu.
\end{abstract}

Keywords: Spatial orientation, navigation aids, small-screen-device.

\section{Introduction}

Recent studies examining the usability of small screen devices, as e.g. the mobile phone, show that users have considerable difficulties to navigate through the device menu [1,2]. Users get lost in a menu system, without knowing where they are, where to go next, and how to get back to previous navigation routes or known parts in the menu $[2,3,4]$. This especially applies for menus implemented in small screen devices, because of the restricted screen space, where users only see parts of the menu they have to navigate through $(2,5)$. On the one hand, the hierarchical menu structure, still the most common form of interaction in communication technology devices, is advantageous as the functions are organized in smaller groups, thus rationing the multitude of options and keeping the information manageable. On the other hand, hierarchical menus also hold great potential for confusions and disorientation when menu structures are complex $[4,5,6,7,8]$. The screen only provides the functions currently available with only little information given about previous or subsequent menu levels. As depth of the phones' menu continuously increases with increasing number of functionalities, still more function labels intervene along the path and seduce the users to 
take the wrong turnoff in the menu, if not being prevented from by excellent verbal memory abilities $[2,6]$. Users need to remember which functions they have already selected during the search and where they are located within menu hierarchy. The memorizing of salient features on the route supports the development of an adequate mental representation of the system structure. If short-term memory's capacity is exceeded, disorientation in the menu occurs, accompanied by additional meandering in search for the targeted function. Users report to loose their bearings in the menu, and, subsequently delve into distraction $[2,3,4]$.

A considerable number of studies underline the importance of spatial orientation processes for the proper handling of technical devices $[4,5,9,10,11,12,13]$, especially small screen devices $[1,2,6]$. When interacting with complex menu structures, users need to build up a mental representation of the spatial structure of that menu in order to orient themselves. The knowledge of how a menu is spatially structured guides users in their search through different levels in different menu depths. Theoretical concepts of spatial orientation $[14,15]$ assume that orientation includes three major spatial knowledge types: route knowledge (the knowledge of the sequence of actions required to get from one point to another), landmark knowledge (representing salient features on the route), and survey knowledge (reflecting the overall structure and providing an overview of locations and routes in space). The concept had been successfully transferred to the navigation through different forms of hypertext $[16,17,18]$, also for PDAs $[1,19,20]$, and for mobile phones $[2,6,8]$. The navigation performance was superior when users had built up survey knowledge (hierarchical nature of the menu), route knowledge (which route to take through the menu), and also landmark knowledge (at which crossings to turn 'left' or 'right'). Spatial abilities specifically advantage navigation by developing a mental representation of the systems' structure $[2,21,22,23]$, which helps users to harmonize their relative menu and to keep the menu structure in mind while navigating through the system.

Yet, an increasing number of studies have been dealing with the usability of computer interfaces and small screen devices for older adults [1,2,3,4,11,19,20,21], addressing the impact of the demographic change. Only few studies considered the difficulties children experience $[8,24,25,26]$ when using mobile devices. Though the investigation of children is of specific interest. Children are an increasingly important user group of small screen devices, and therefore need usable designs. Also, children are supposed to easily master the interaction with technical devices and to understand the mode of operation of those devices much faster by virtue of their early contact with technology. Additionally, children's fascination for explorative and inquisitive activities is well known. According to recent studies [25,26] evidence is given that children showed considerable performance losses in suboptimal interface designs.

The present study examines the utility of two navigation aids, which were implemented into the phones' display. One type provides survey knowledge and the other landmark knowledge. In order to learn how user characteristics interact with navigation in mobile phones, children and younger adults were examined and verbal memory and spatial abilities were surveyed. The first question to be answered is if any navigation aid implemented in the small screen of a miniature device is helpful at all. The critical argument in this context refers to screen space restrictions and the limited amount of information to be displayed at a time. Any additional information on small screens also bears the danger of causing visual overload, possibly interfering with the visual 
encoding process and affecting information processing [27). As additional information load in the small display should be held as low as possible, maximizing the effectiveness of information access, the adding of information could also disadvantage performance by causing visual load. The second question is if there are differences respecting the type of information which is delivered by the navigation aid. Here, it is of interest if children, which have lower cognitive abilities due to their developmental status, can process the spatial information delivered by navigation aids.

\section{Method}

\subsection{Independent and Dependent Variables}

Two independent variables were examined. The first independent variable was the type of navigation aid. Two different navigation aids were implemented into a computer simulation of a real mobile phone. In one condition, the interface showed the name of the current category, as well as a list of its contents. This aid delivers mainly landmark knowledge without spatial information about the menu structure (Fig. 1, center). The other interface was identical to the first except that it showed the parents and parent-parents of the current of the category and that sub-categories were indented to emphasize the hierarchical structure (Fig. 1, right). This aid contained spatial cues, providing survey knowledge (structure of the menu). In addition, a control condition was examined, in which no navigation aid was given. Instead, the original interface was presented (Fig. 1, left). In each of the three cases shown in Figure 1, the top display is a higher level version of the bottom display.

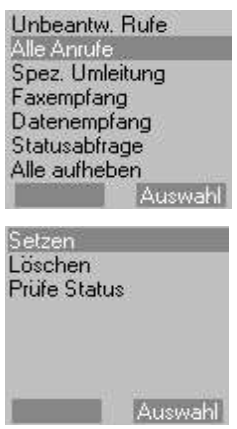

No aid
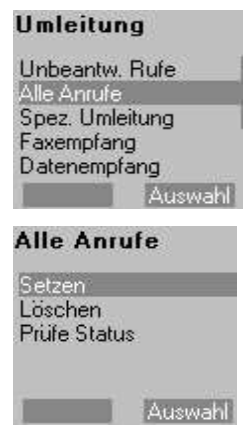

aid with landmark knowledge

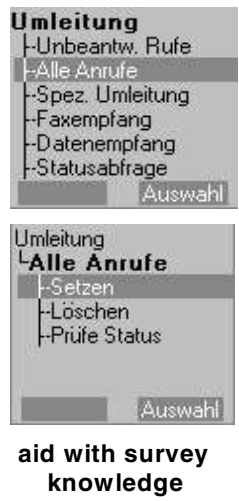

Fig. 1. Visualizations of the three different interface conditions of the mobile phone

The second independent variable was users' age, comparing the performance of children, and younger adults. In addition, verbal memory and spatial abilities were psychometrically determined and treated as between subject variables, possibly affecting navigation performance and interacting with the navigation aids.

Dependent variables were the effectiveness and efficiency of navigation [28]. For the task effectiveness, the number of successfully solved tasks (within the time limit 
of five minutes per task) was measured. A maximum of eight tasks (four tasks solved twice) were to be completed. For efficiency, three different measures were collected: The time needed to process the tasks was surveyed. Also, hierarchical returns to higher levels in menu hierarchy were analyzed. Returns in menu hierarchy occur when users in the belief of having taken the wrong path go back to a known menu position, consequently re-orientating themselves. In addition, returns to the top were determined. This measure was assumed to reflect utter disorientation, as users had to re-orientate by returning to the top menu level, beginning from scratch.

\subsection{Experimental Tasks}

Four typical and frequently used mobile phone tasks were selected. In order to determine learnability effects, the tasks had to be solved twice consecutively. The order of tasks in the two trials was held constant over participants. In total, a minimum of 47 steps was necessary to solve the four tasks. Participants had to

- Call a number (11 keystrokes).

- Hide one's own number when calling someone (14 keystrokes).

- Send a text message (11 keystrokes; the message was already provided and only had to be sent when participants had reached the adequate point in the menu).

- Make a call divert to the mailbox (11 keystrokes).

\subsection{Participants}

48 participants volunteered to take part. 24 children (nine boys, 11 girls), aged 9 - 10 years $(\mathrm{M}=9.5 ; \mathrm{SD}=0.8)$ of age and 24 young adults (12 males, 12 females), aged 23 - 31 years $(M=24.9 ; S D=2.9))$. The children were pupils in their fourth school year; the adults were students of different academic fields. Participants' experience with mobile phones and other technical devices was studied as well as the reported ease of using them. They reported if and how often they use a mobile phone and a PC, using a 5 -point scale $(1=$ several times per day, $2=$ once per day, $3=$ once or twice a week, 4 $=$ once or twice per month and $5=$ less than once or twice a month). Furthermore, they rated the ease of using these devices, using a scale ( $1=$ the usage is easy, $2=$ the usage is rather easy, $3=$ the usage is rather difficult and $4=$ the usage is difficult). Finally, participants indicated their interest in technology, using a 4-point scale ( $1=$ low interest; 2 = rather low interest, $3=$ rather high interest, $4=$ high interest).

\subsection{Assessing User Characteristics Interacting with Navigation Performance}

It was a major aim to learn how age groups were interacting with the mobile phone and, which user characteristics might be crucial for navigation performance. Therefore, participants were surveyed regarding spatial ability and verbal memory capacity; both cognitive abilities help to built up a mental representation of the system structure. For the children, two subtests of the HAWIK-R were carried out. In the spatial ability test ("Mosaic Test") the experimenter showed the child a picture (Fig. 2, left) and the child's task was to reproduce the picture using cubes having different patterns on each of the sides (max. 26 points). The test on short-term memory required the children to verbally repeat a row of numbers read aloud by the experimenter, which 
had to be reproduced directly after. The children were given two trials to correctly reproduce each row (max: 14 points). For the adult group, spatial abilities were assessed with the paper-folding test [29]). Each of the twenty items includes successive drawings of two or three folds made in a square sheet of paper. The final drawing shows a hole punched in the folded paper. Participants had to mentally rotate the paper from the folded into the fully opened form and to indicate which of a number of possibilities shows the correct drawing. The 20 items had to be solved within $180 \mathrm{~s}$. In Figure 2 (right), an example item of the paper-folding test is given. To assess memory ability, the verbal memory test [6] was used.15 Turkish words (unknown to Germans) were presented in succession for $3 \mathrm{sec}$, each. Directly after, users had to recognize the target items among three distractors, each being phonologically or visually similar. The maximum score was 15 .
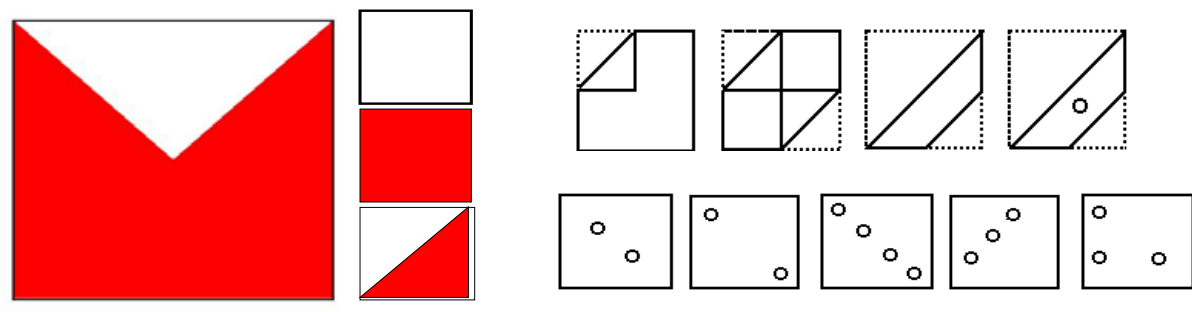

Fig. 2. Left: the Mosaic-Test to assess children's spatial visualization ability [30]; right: Item example of the Paperfolding test to assess adults' spatial visualization ability [29]

\subsection{User Characteristics of Participants}

In this section, the experience with technical devices, and the reported ease of using them are focused at. Also, the rated general interest in technology is illustrated. Furthermore, the participants' verbal memory and spatial abilities are described. It is of interest, if these variables are modulated by age or the gender of participants.

Technical experience. Users showed a high experience with technical devices, however, significant age differences were present $(\mathrm{F}(1,40)=18.8 ; \mathrm{p}<0.05$. In contrast to the adults which used mobile phones and PCs several times a day (phone: $\mathrm{M}=1.3 ; \mathrm{SD}=$ $0.5 ; \mathrm{PC}: \mathrm{M}=1.4 ; \mathrm{SD}=.48)$, the children used the mobile phone $(\mathrm{M}=3.1 ; \mathrm{SD}=1.1)$ and the $\mathrm{PC}(\mathrm{M}=2.2$; $\mathrm{SD}=1.3)$ less frequently. The interest in technology was generally high, but also showed significant age differences $(F(1,40)=15.8 ; \mathrm{p}<0.05)$. Interestingly, the children's interest in technology $(\mathrm{M}=3.5 ; \mathrm{SD}=0.6)$ was higher compared to the adults $(\mathrm{M}=2.8 ; \mathrm{SD}=0.7)$. Also, gender differences were revealed $(\mathrm{F}(1,40)=13.1 ; \mathrm{p}<0.05)$. Independently of age, male users reported a higher interest in technology $(M=3.5 ; \mathrm{SD}=0.8)$ than did female participants $(\mathrm{M}=2.9 ; \mathrm{SD}=0.8)$.

Verbal memory. First, outcomes in children's verbal memory are reported. From the 14 points that could be attained at the most, the children reached, on average, 5.5 points $(\mathrm{SD}=1.4)$. Apparently, the memorizing and recall of the digits was quite difficult for them, as none of the children was able to reach the maximum score. For the adults, the performance equaled 13.1 ( $\mathrm{SD}=1.9$ ) points (out of 15). Gender differences were not present, neither in the children nor the adult group. 
Spatial visualization abilities. The children differed considerably with respect to the extent of spatial abilities. The lowest performance in the mosaic test was 5 points, however, there were also children, who reached the maximum score of 26 points $(\mathrm{M}=$ 15.2; $\mathrm{SD}=5.1)$. With respect to the performance of the adults, the spectrum of correct answers ranged between 9 and 19 (out of 20), reaching a mean performance of 13.3 $(\mathrm{SD}=3.1)$. No gender differences in spatial visualization ability were found.

\subsection{Apparatus and Materials}

The utility of navigation aids is a rather generic question, which is quite independent of the device type. A simulation of a real mobile phone was used (Siemens S45). None of participants had used the specific model before. As individual navigation routes had to be analyzed in detail, the phone was simulated as software solution, run on a PC, and displayed on a touch screen (Iiyama TXA3841). The display corresponded to the original size, but the phone's chassis and the keys were enlarged enabling easy operation with the finger on the touch screen. Moreover, a logging software tool was developed, which logged any user interaction with the system. By this, the number and type of keys used, the functions selected, and the individual navigation routes taken through the menu could be reconstructed in detail. In order to assure a comfortable body posture, participants sat on a table and worked on the touch screen fixed to the edge of the table (Figure 3).

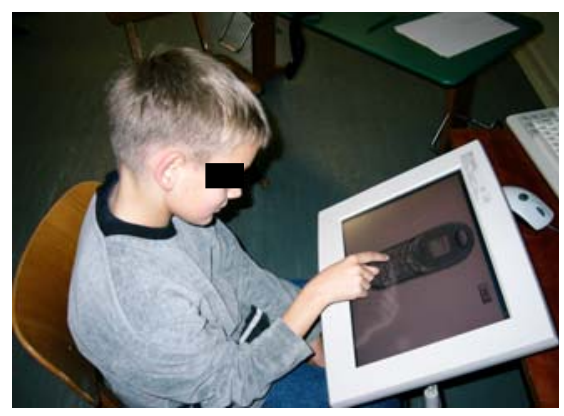

Fig. 3. Photo of a child using the simulated mobile phone on the touch screen

\subsection{Design and Procedure}

The study was based on a two-factorial design. Both main factors (navigation aid and age) were treated as between-subject variables. Gender was balanced. Spatial visualization and verbal memory abilities were treated as between subject variables.

In the beginning of the experiment, participants' technical experience was assessed and verbal memory and spatial abilities were determined. Then, the nine tasks were to be solved twice, in order to determine learnability effects. A time limit of five minutes per task was set (the appropriateness of the limit was tested in previous studies). A fast and thorough working style was instructed. If a task was solved successfully, a 'Congratulations' - message appeared on the display. To avoid any biases, the aids were neither explained nor mentioned. It was instructed that the experiment was 
concerned with the usability of mobile phones. All participants were told that -in case of a very difficult task- they should feel free to "give up", in order not to overly burden them. However, none of participants did so, but rather enjoyed the experiment. Depending on the individual working speed, the experiment lasted about 60 minutes.

\section{Results}

The results were analyzed by multivariate analyses of variance assessing effects of the type of navigation aid and age on effectiveness (tasks solved) and efficiency (time, hierarchical returns and returns to the top). The significance of the omnibus F-Tests was taken from Pillai values, followed by the description of the single F-tests. Also, learnability effects were analyzed, determining if performance improved from the first to the second trial). A final analysis was concerned with the impact of user characteristics for performance. The level of significance was set at $p<0.05$.

Regarding the analysis of task efficiency, two strategies can be considered: One strategy only includes users that successfully accomplished the tasks; the other one includes all participants, independently from their tasks' success. Both strategies are basically "sensible". On a first sight, the selection of successful task performers for further analysis seems to be advantageous as their results can be directly related to effectiveness. However, if only a rather small proportion of participants were able to solve a certain task, only small and unequal samples would have entered statistical analyses. Moreover, from an ergonomic point of view, it is more insightful to learn about the ergonomic shortcomings and navigation difficulties and to consider all user actions -- even if users failed at the end. Analyses showed that result patterns for "task solvers" and "non-solvers" were quite similar. Therefore, task efficiency was statistically analyzed comprising the total group.

\subsection{Effects of Navigation Aids}

A first analysis was concerned with the different types of navigation aids. MANOVA analyses revealed a significant omnibus effect $(F(4,80)=2.9 ; \mathrm{p}<0.05)$. On the single F-test level, the main effect of navigation aid was significant for the effectiveness $(\mathrm{F}(2,42)=5.4 ; \mathrm{p}<0.05)$, the returns in menu hierarchy $(\mathrm{F}(2,42)=3.6 ; \mathrm{p}<0.05)$, and the returns to the top. The descriptive outcomes can be seen in Figure 4 .

From figure 4 it can be seen that the lowest performance was given in the condition where no navigation aid was given (white bars). The disadvantage of having no aid is especially prominent in the two disorientation measures, the returns in menu hierarchy and the returns to the top. However, the utility of the two different aids turned out to be different. The aid providing landmark knowledge did not differ from the no-aid condition, yielding about the same (low) level of performance, what is -again- very pronounced in the disorientation measures. Having the aid providing landmark knowledge as support, users spent $14 \mathrm{~min}$ on the tasks and stepped, on average, 61.5 times (SD = 37) back to higher levels in the menu. In contrast, supported by the aid delivering survey knowledge, this happened about half as often $(M=29.9 ; \mathrm{SD}=23)$. The difference yielded a significant effect $(F(2,42)=3.6 ; \mathrm{p}<0.05)$. The same pattern can be seen in the number of returns to the top. In the landmark aid, users had to re-enter the menu, 6.6 times $(\mathrm{SD}=5.3)$, while it occurred significantly less often $(\mathrm{M}=2.1 ; \mathrm{SD}=2.3)$ in the navigation aid delivering survey knowledge $(\mathrm{F}(2,42)=4.2 ; \mathrm{p}<0.05)$. 

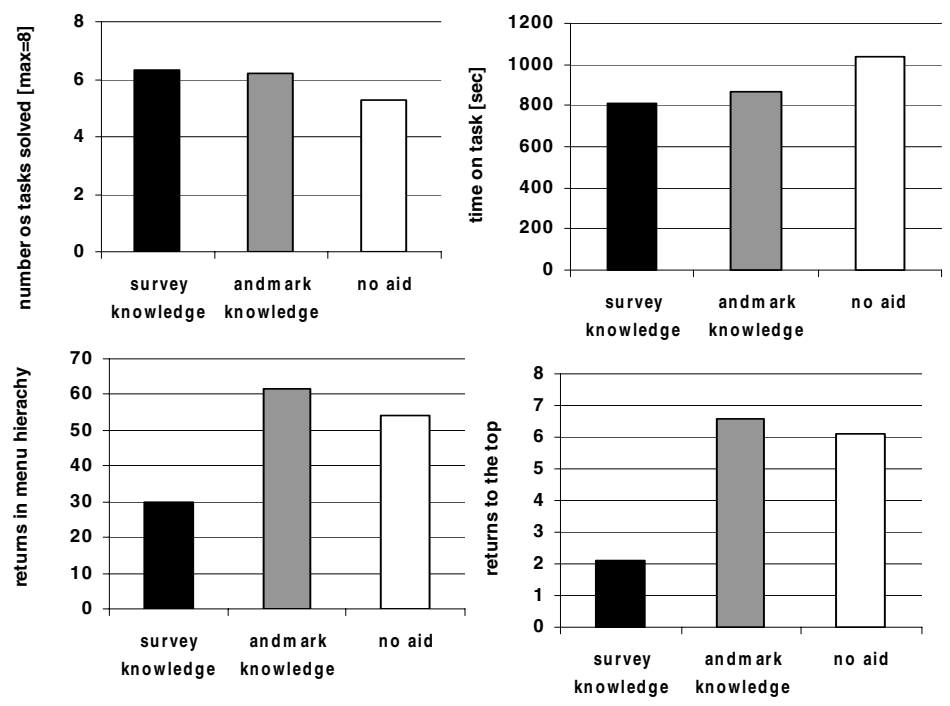

Fig. 4. Effects of navigation aid. Upper left: effectiveness; upper right: time on task; lower left: returns in menu hierarchy; lower right: returns to the top.

\subsection{Effects of Age}

Next, the performance of the children compared to the adults is focused. With respect to task effectiveness, significant $(\mathrm{F}(1,42)=86.5 ; \mathrm{p}<0.05)$ age effects were found. Of the eight tasks, children solved, on average, 4.2 tasks $(\mathrm{SD}=1.7)$ successfully, and the adults 7.6 tasks $(\mathrm{SD}=0.8)$. The age differences were even more prominent regarding efficiency: The adults needed, on average, 6 minutes $(\mathrm{SD}=256 \mathrm{~s})$ to process all tasks, while the children needed 24 minutes $(S D=416 \mathrm{~s} ; \mathrm{F}(1,42)=118.5 ; \mathrm{p}<0.05)$. When navigating through the menu, adults made 21.4 hierarchical returns $(\mathrm{SD}=16.8)$, and, on average, 1.8 returns to the top $(\mathrm{SD}=2.2$ ). Again, children's efficiency was much lower, carrying out more than three times as many hierarchical steps back $(\mathrm{M}=75.6$; $\mathrm{SD}=49)$ with, on average, more than four times as many returns to the top $(\mathrm{M}=8.1$; $\mathrm{SD}=6.9)$. Age differences in both, hierarchical steps back $(\mathrm{F}(1,42)=28.9 ; \mathrm{p}<0.05)$
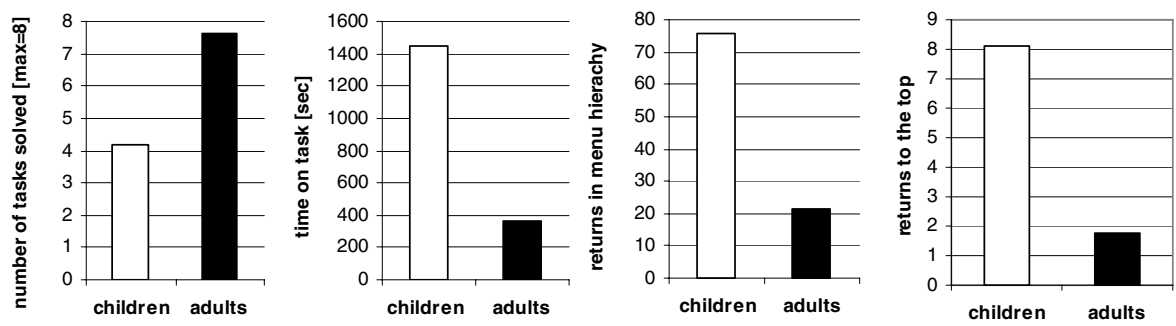

Fig. 5. Effects of age on all dependent measures 
and returns to the top $(\mathrm{F}(1,42)=21.3 ; \mathrm{p}<0.05)$ yielded statistical significance. In Figure 5, age differences are pictured for all dependent measures.

The interaction of both variables, the type of navigation aid and users' age had shown to be significant $(\mathrm{F}(8,80)=2.1 ; \mathrm{p}<0.05)$. The nature of the co-acting of navigation aid and age becomes evident from Figure 6.
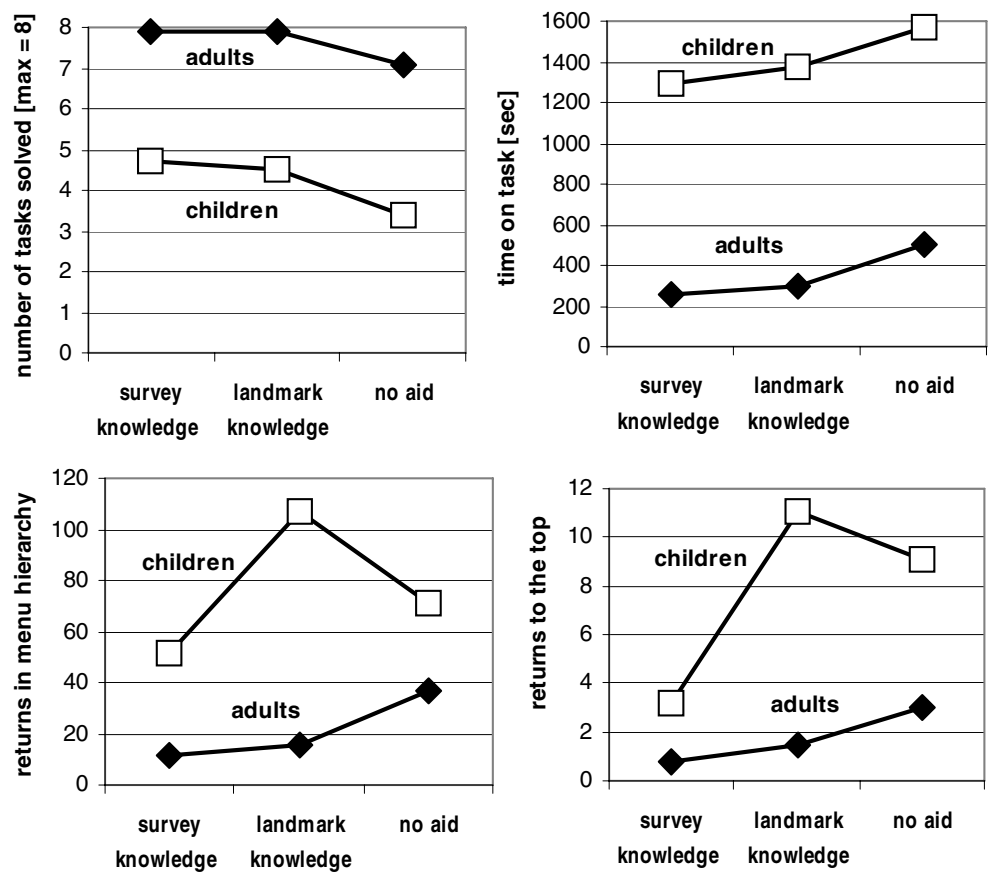

Fig. 6. Interacting effects between age and navigation aid for all dependent measures

The interaction mainly stems from the disorientation measures (returns in menu hierarchy, and returns to the top). While the aid with the survey knowledge yielded advantageous effects on the performance in both age groups, the aid delivering landmark knowledge showed detrimental effects for the children, as taken from the considerably higher detouring in the menu. With respect to the number of returns in menu hierarchy, the disadvantage of the landmark knowledge aid compared to the survey knowledge aid for the children was $55 \%$ and, for the number of returns to the top even $70 \%$.

\subsection{Learnability Effects}

Can users improve their performance in the second trial? With respect to task effectiveness significant improvements were found (effectiveness: $F(1,36)=4.4 ; p<0.05$ ), however, not differing between the navigation aids conditions (Figure7). In the first trial, 2.9 ( $\mathrm{SD}=1.1)$ of the four tasks were solved and 3.1 tasks $(\mathrm{SD}=1.1)$ in the second. Looking at the time on task, also significant learnability effects were detected $(\mathrm{F}(1,36)=22.3 ; \mathrm{p}<0.05)$. However, learnability differed between conditions, revealing a significant interacting effect $(\mathrm{F}(2,36)=3.3 ; \mathrm{p}<0.05$, Figure 7$)$. 

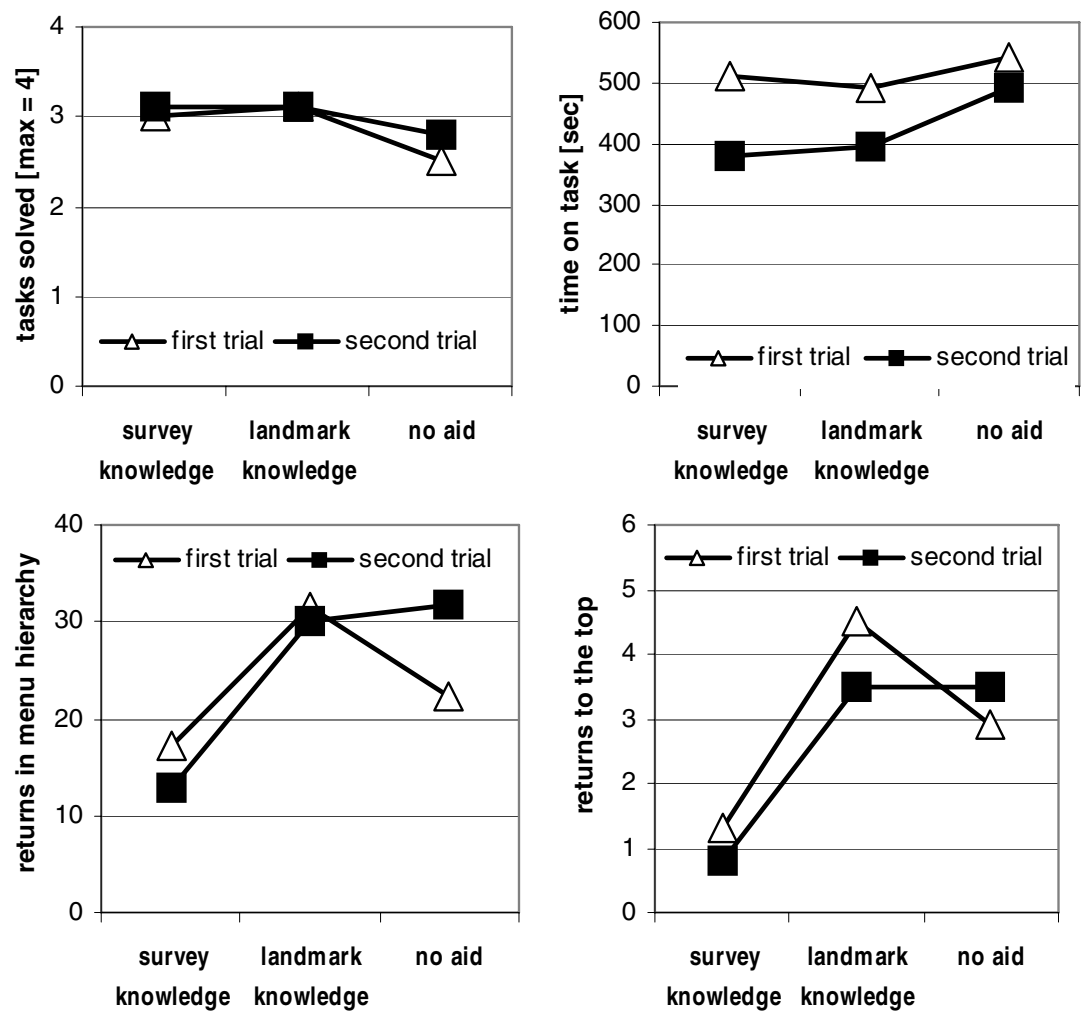

Fig. 7. Learnability effects in the three experimental conditions

From Figure 7 it can seen that, learnability effects were mainly limited to the time on task, showing that participants were considerably faster processing the phone tasks a second time. For the disorientation measures, performance did not significantly improve from the first to the second trial. Detailed analyses showed that the reason for the non-significant learnability effect is the low performance in the condition in which no aid was present. Here, the performance in the second trial was even lower than in the first trial. This negative learnability effect in the control condition corroborates that participants have major difficulties developing a mental representation of the menu without any navigation aid.

\subsection{Impact of User Characteristics on Performance Outcomes}

Finally it is analyzed whether there are crucial user characteristics to be considered which influence the navigation performance. Also it is of interest if user characteristics interact with the utility of the navigation aids. Answering the second question first, no interacting effect between user characteristics and the utility of navigation aids were found. Thus, we can assume that the benefit by the aids, and specifically the benefit of the aid providing survey knowledge is universal and thus can be recommended for a 
broad variety of users. However, there were significant correlations between user characteristics and navigation performance. For the adult group, spatial visualization ability was significantly correlated with the time needed to process the tasks $(r=-.57 ; \mathrm{p}<0.05)$, the number of returns in menu hierarchy $(\mathrm{r}=-.51 ; \mathrm{p}<0.05)$, and the returns to the top $(\mathrm{r}=-.47 ; \mathrm{p}<0.05)$. Thus, persons with high spatial abilities are considerably advantaged. Memory abilities and technical experience did not show a significant impact on performance. For the children group, task effectiveness turned out to be significantly related to the frequency of using a mobile phone $(\mathrm{r}=-.39 ; \mathrm{p}<0.05)$ and the ease of using it $(\mathrm{r}=-.44 ; \mathrm{p}<0.05)$. Also, time on task showed significant correlations with the frequency of using a mobile phone $(\mathrm{r}=-.39 ; \mathrm{p}<0.05)$, as well as a $\mathrm{PC}(\mathrm{r}=-.44 ; \mathrm{p}<0.05)$, and also with the ease of using the mobile phone $(\mathrm{r}=-.4 ; \mathrm{p}<0.05)$ and the $\mathrm{PC}(\mathrm{r}=-.4 .2 ; \mathrm{p}<0.05)$. Thus, children with a high technical experience are advantaged in menu navigation. The level of verbal memory and spatial visualization, did not affect children's menu navigation.

\section{Discussion and Conclusion}

As disorientation is a frequent problem when using mobile small-screen devices with a hierarchical menu [e.g. 1,2,3,20], the utility of navigation aids implemented in the menu of a mobile phone menu was under study. The navigation aids contained different types of spatial knowledge. Based on the theoretical framework of spatial orientation $[14,14]$, two types of spatial knowledge very varied within the navigation aids. One type represented an aid mainly containing landmark knowledge, which tells users salient features on the navigation route. The other type delivered spatial information and survey knowledge, which informs users about the menu structure and providing an overview of locations and routes in space. However, as any additional information on the restricted screens may also cause visual overload and interfere with the visual encoding process [27], affecting information processing, a control condition was examine $122 \mathrm{~d}$, in which no aid was given. Here, participants used the original display design of the phone.

The results clearly showed that the handling of a common mobile phone is not easy to accomplish, but rather represents a high cognitive challenge for users. Especially the 9-10 years olds showed considerable difficulties to master the four common phone tasks on a common mobile phone. Tasks effectiveness ranged only at about $50 \%$, accompanied by a huge number of detouring routes. Children executed many returns in menu hierarchy, re-orientating themselves, even returning frequently to the top menu level, beginning from scratch. Also the young adults, students, bright and technologyprone, which do not represent the average user, but a kind of "best case" user group, did not show a "perfect" performance, solving all the tasks without barriers. Taken from their detouring routes, they also experienced disorientation in the menu. Facing the increasing penetration of small screen devices in all parts of daily life, and considering the growing user diversity, this corroborates the enormous impact of usable designs. It must be concluded that current small screen devices are -- cognitively -challenging to use. This is true for young adults, but it is even more valid for children, which -due to their developmental status- can be categorized as "weaker" users $[8,24,25,26]$. The fact that both navigation aids advantaged users' performance in the 
menu shows that the disorientation problem in small screen device menus can be basically relieved. It is an important finding that useful and usable designs do not necessarily require huge sums of additional costs with respect to the implementation in state-of-the-art mobile design. As shown here, very small software modifications can be very effective and in an ergonomic sense, successful.

The answer to the question which of both navigation aids is more helpful for users reveals both, universal and differential aspects to be considered for usable interface designs. The aid which delivered structural information and survey knowledge showed to have a universal benefit, advantaging performance for both, the children and the adults. Task success was higher and the detouring in the menu was distinctly reduced, taken from the smaller number of detour steps and returns to higher levels in menu hierarchy. It is assumed that the nature of the strong benefit by the survey knowledge lies in the fact that users are supported in constructing a proper mental representation of the systems' structure [4,9,12,21,23]. They are informed about their relative position in the menu and can keep the menu structure in mind while navigating through the system. Thus, by having an appropriate model and a structural concept of the mental 'room' that has to be navigated through, performance is distinctly increased. In the mobile phone, where the overall structure of the menu is not transparent, and the screen size is very limited this is even more crucial.

The aid delivering mainly landmark knowledge (showing the name of the current category and a list of its contents), though, turned out to be age-sensitive. While young adults simply showed a somewhat lower performance (compared to the aid delivering survey knowledge), the children had extremely difficulties handling the aid providing landmark knowledge. Apparently, the kids were not able to capitalize on the landmark information when searching for a specific function. In the contrary, their navigation was impeded by this aid, as taken from the fact that the detouring in the menu was dramatically increased when having the landmark aid as support.

The findings presented here do have implications for the design of mobile phones in general. First, it is important to learn that the way the information and menu of a system are structured seemed not to be fully transparent to children - even if they are used to working with programs such as Windows Explorer ${ }^{\circledR}$, which is organized in the same fashion as our sample. Therefore, the constant visualization of the menu structure is of unobtrusive assistance. Another way of overcoming disorientation problems associated with hierarchical menu structures [31] is to use only one long alphabetical list of functions, where users can search by initial letters. This was evaluated with students. It is to be questioned whether this really helps users less experienced with mobiles as they often have no idea of the functions' naming in the menu and simple recognition of functions and categories - even though far from trivial should be easier than active recall of the right term for a specific function.

From the outcomes of this study both, important knowledge about user groups as well as practical design recommendations can be derived.

- Even the children, which are broadly assumed to easily master the usage of small screen device, have serious difficulties to handle small screen devices.

- Applying a navigation aid in the small screen which informs users about the spatial structure of the menu can compensate for these difficulties.

- Designers and manufactures should take usability issues serious and implement spatial cues as navigation aids into the small screen of mobile devices. 
- The utility of this kind of navigation aid is not restricted to phones, but can be easily transferred to any small screen device with a hierarchical menu, as MP3-Players, eHealth devices for medical care, wrist watches, fax machines, printers, banking and ticket machines or mobile gaming devices etc.

Future studies will have to extend this research by examining navigation aids that are not only visual, but use auditory and sonically enhanced navigational cues [32,22].

Acknowledgments. The author thanks Susanne Bay for valuable contributions to this research. Thanks also to Philipp Brauner, Eugen Yu, Thomas Michel, Luisa Bremen, Judith Strenk, and Fabia Tucht for their research support.

\section{References}

1. Goodman, J., Gray, P., Khammampad, K., Brewster, S.: Using landmarks to support older people in navigation. In: Brewster, S., Dunlop, M.D. (eds.) Mobile HCI 2004. LNCS, vol. 3160, pp. 38-48. Springer, Heidelberg (2004)

2. Ziefle, M., Bay, S.: How to overcome disorientation in mobile phone menus: A comparison of two different types of navigation aids. Human Computer Interaction 21(4), 393-432 (2006)

3. Arning, K., Ziefle, M.: Barriers of information access in small screen device applications: The relevance of user characteristics for a transgenerational design. In: Stephanidis, C., Pieper, M. (eds.) ERCIM Ws UI4ALL 2006. LNCS, vol. 4397, pp. 117-136. Springer, Heidelberg (2007)

4. Lin, D.-Y.: Hypertext for the aged. Computers in Human Behaviour 19(2), 201-209 (2003)

5. Edwards, D., Hardmann, L.: Lost in Hyperspace. Cognitive Mapping and Navigation in a Hypertext Environment. In: McAleese, R. (ed.) Hyperspace: Theory into practice, pp. 105125. Intellect limited, Oxford (1989)

6. Bay, S., Ziefle, M.: Design for all: User characteristics to be considered for the design of phones with hierarchical menu structures. In: Luczak, H., Zink, K.J. (eds.) Human Factors in Organizational Design and Management, pp. 503-508. IEA Press, Santa Monica (2003)

7. Billingsley, P.A.: Navigation through hierarchical menu structures: Does it help to have a map? In: Proc Human Factors Society 26th Annual Meeting, pp. 103-107 (1982)

8. Bay, S., Ziefle, M.: Children using cellular phones. The effects of shortcomings in user interface design. Human Factors 47(1), 158-168 (2005)

9. Downing, R., Moore, J., Brown, S.: The effects of spatial visualization and domain expertise on information seeking. Computers in Human Behaviour 21, 195-209 (2005)

10. Norman, K.: Spatial visualization - a gateway to computer-based technology. Journal of Special Education Technology 12(3), 195-206 (1994)

11. Pak, R., Czaja, S., Sharit, J., Rogers, W., Fisk, A.: The role of spatial abilities and age in performance in an auditory computer navigation task. Computers in Human Behaviour 24(6), 3045-3051 (2008)

12. Allen, G.L.: Human Spatial memory. Remembering where. LEA, Mahwah, NJ (2004)

13. Westerman, S.J.: Individual differences in the use of command line and menu computer interfaces. Int. J. of Human Computer Interaction 9(2), 183-198 (1997)

14. Thorndyke, P.W., Goldin, S.: Spatial learning and reasoning skill. In: Pick, H.L., Acredolo, L.P. (eds.) Spatial orientation, pp. 195-217. Plenum, New York (1983) 
15. Thorndyke, P.W., Hayes-Roth, B.H.: Differences in spatial knowledge acquired from maps and navigation. Cognitive Psychology 14, 560-589 (1982)

16. McDonald, J., Stevenson, R.: Navigation in Hyperspace: An evaluation of the effects of navigational tools and subject matter expertise on browsing and information retrieval in hypertext. Interacting with Computers 10, 129-142 (1998)

17. Vicente, K.J., Hayes, B.C., Williges, R.C.: Assaying and isolating individual differences in searching a hierarchical files system. Human Factors 29(3), 349-359 (1987)

18. Kim, H., Hirtle, S.C.: Spatial metaphors and disorientation in hypertext browsing. Behaviour and Information Technology 14(4), 239-250 (1995)

19. Arning, K., Ziefle, M.: Understanding differences in PDA acceptance and performance. Computers in Human Behaviour 23(6), 2904-2927 (2007)

20. Arning, K., Ziefle, M.: Cognitive and personal predictors for PDA navigation performance. Behaviour and Information Technology (2009), doi:10.1080/01449290701679395

21. Ziefle, M., Bay, S.: Mental models of a cellular phone menu. Comparing older and younger novice users. In: Brewster, S., Dunlop, M.D. (eds.) Mobile HCI 2004. LNCS, vol. 3160, pp. 25-37. Springer, Heidelberg (2004)

22. Mayer, R.E.: Models for Understanding. Review of Educational Research 59(1), 43-64 (1986)

23. Sein, M.K., Olfman, L., Bostrom, R.P., Davies, S.A.: Visualisation ability as a prediction of user learning success. Int. J. Man-Machine-Studies 39, 599-620 (1993)

24. Ziefle, M., Bay, S.: Transgenerational Designs in Mobile Technology. In: Lumsden, J. (ed.) Handbook of Research on User Interface Design and Evaluation for Mobile Technology, pp. 122-140. IGI Global, Hershey (2008)

25. Ziefle, M., Bay, S., Schwade, A.: On keys' meanings and modes: The impact of navigation key solutions on children's efficiency using a mobile phone. Behaviour and Information Technology 25(5), 413-431 (2006)

26. Bay, S., Ziefle, M.: Landmarks or surveys? The impact of different instructions on children's performance in hierarchical menu structures. Computers in Human Behaviour 24(3), 1246-1274 (2008)

27. Bay, S., Ziefle, M.: Effects of menu foresight on information access in small screen devices. In: 48th Annual Meeting of the Human Factors and Ergonomic Society, pp. 18411845. Human Factors Society, Santa Monica (2004)

28. EN ISO 9241-11, Ergonomic requirements for office work with visual display terminals. Part 11: Guidance on usability, Beuth, Berlin (1997)

29. Ekstrom, R.B., French, J.W., Harman, H.H., Dermen, D.: Manual for the Kit of FactorReferenced Cognitive Tests. Educational Testing Service, Princeton (1976)

30. Tewes, U.: Hamburg-Wechsler-Intelligence Test. Huber, Bern, Switzerland (1983)

31. Marsden, G., Jones, M.: Ubiquitous computing and cellular handset interfaces - are menus the best way forward? South African Computer Journal 28, 67-76 (2002)

32. Chittaro, L., Burigat, S.: Augmenting audio messages with visual directions in mobile guides: an evaluation of three approaches. In: Proceedings of the 7th international conference on Human computer interaction with mobile devices \& services, pp. 107-114 (2005)

33. Brewster, S.: Overcoming the Lack of Screen Space on Mobile Computers. Personal and Ubiquitious Computing 6(3), 188-205 (2002) 